\title{
Morphological Changes of Cisplatin-resistant Human Breast Cancer MCF-7 Cell Line
}

\author{
Nanda Ayu Puspita, ${ }^{1,2}$ Amy Bedford ${ }^{3}$ \\ ${ }^{1}$ Departement of Biochemistry, Faculty Medicine, Syiah Kuala University, Banda Aceh, Indonesia \\ ${ }^{2}$ Biomedical Research Centre, University of Salford, The Crescent, Salford, United Kingdom \\ ${ }^{3}$ Faculty of Biology, Medicine and Health, University of Manchester, Oxford Road, Manchester, United Kingdom
}

\begin{abstract}
Objective: To evaluate morphological changes of breast cancer cell line MCF-7 following the development of acquired resistance towards cisplatin. Resistance towards anticancer agents still plays an important role in the breast cancer chemotherapy failure.

Methods: The cisplatin resistant subline MCF-7/CisR was developed in-vitro by cultivating the parental cell line cisplatin-sensitive MCF-7 with raising concentration of cisplatin (from 0.01 to $2.3 \mu \mathrm{M}$ ) for four months. The cell morphology was observed by giemsa staining.

Results: The resistance was shown by the increase of $\mathrm{IC}_{50}$ by 9 folds on MCF-7/CisR compared to the initial $\mathrm{IC}_{50}$ value of MCF-7. The change in cisplatin cytotoxic potency on the resistant cell line was accompanied by the morphological modification, including the enlargement of cell size, the increase of nucleus and cytoplasm ratio, and the increase of the number of microvesicular and cytoplasmic granules.
\end{abstract}

Received:

December 14, 2016

Conclusions: This result has supported the underlying mechanism of cisplatin resistance, including the ability of the cells to decrease intracellular

Revised: cisplatin concentration and repair DNA damage effects.

February 17, 2017

Accepted:

February 27, 2017

Keywords: Drug resistance, human breast cancer, cisplatin, MCF-7

pISSN: 2302-1381; eISSN: 2338-4506; http://doi.org/10.15850/ijihs.v5n1.960

IJIHS. 2017;5(1):8-14

\section{Introduction}

Breast cancer is known as the most common cancer in women. ${ }^{1,2}$ In the developed world, for example, in the United Kingdom, around 150 new cases are diagnosed every day; 1 in 8 women are predicted to be diagnosed with breast cancer during their lifetime. Meanwhile, in less developed countries, the breast cancerrelated deaths reach a significant number, which is more than $50 \%$ of the diagnosed cases. ${ }^{3}$ In combating breast cancer, the modality of therapy can include surgery, chemotherapy, and radiotherapy. ${ }^{1-3}$ For a systemic approach, chemotherapy is counted as a key strategy to treat breast cancer due to eliminate residual

\section{Correspondence:}

Nanda Ayu Puspita, Departement of Biochemistry,

Faculty Medicine, Syiah Kuala University

Jl. T. Nyak Arief Darussalam Banda Aceh 23111, Banda

Aceh, Indonesia

e-mail: n.a.puspita@edu.salford.ac.uk cancer after surgery or possible occurrence of metastatic cells. ${ }^{4}$ However, chemotherapy is frequently accompanied by the occurrence of drug resistance of the cancer cells towards one or multiple drugs which accounts for the failure of the breast cancer treatment. ${ }^{1,3,4}$

Breast cancer cells were notably reported to develop resistance towards chemotherapy agents, including platinum derivatives such as cisplatin. ${ }^{4-6}$ Until recently, the clear mechanism of drug resistance of breast cancer cell is still in debate. ${ }^{4-8}$ Many studies have reported several possible mechanisms which are related to chemo-resistance, including over-expression of p-glycoprotein, alteration of cell cycle arrest and apoptosis, the expression of multidrug resistance-associated protein (MRP). .,6 $^{6}$ Overall, all these molecular mechanisms will result in the drug-resistant phenotype modification, which could be accompanied by changes in proliferation potential and morphological structure. ${ }^{5}$ Therefore, in order to observe the 
characteristics in the early stages of acquired drug-resistance in breast cancer, structure of the cell cancer might provide an initial insight of the development of chemo-resistance.

The aim of this study was to observe the morphological changes on human breast cancer cell line MCF-7 following the in-house development of the subline resistant to cytotoxic effects of cisplatin (MCF-7/CisR).

\section{Methods}

In this study, the cell line used was human breast cancer cell line MCF-7 from ATTC, United Kingdom (UK). The cell culture and other laboratory procedures were conducted in the Biomedical Research Centre (BMC), University of Salford, UK. Culture media and all media supplements were obtained from Lonza, Walkersville, MD. Dimethyl Sulfoxide (DMSO), cisplatin, and thiazolyl blue tetrazolium bromide (MTT salt) were purchased from Sigma-Aldrich, Dorset, UK.

The MCF-7 cell line was cultivated in Dulbecco's Modified Eagle (DMEM) medium supplemented with $10 \%$ (v/v) fetal bovine serum (FBS), $2 \mathrm{mM}$ L-glutamine, $50 \mathrm{IU} / \mathrm{ml}$ penicillin, and $50 \mu \mathrm{g} / \mathrm{ml}$ streptomycin, at 37 ${ }^{0} \mathrm{C}$ in a humidified atmosphere containing $5 \%$ $\mathrm{CO}_{2}$. The cells were subcultured twice a week or after reaching 70-80\% confluence.

The cisplatin resistant variant (MCF-7/ CisR) was developed from the parental line MCF-7 by cultivating the parental cell line with raising concentration of cisplatin (from 0.01 to $2.3 \mu \mathrm{M}$ ). Cisplatin was added twice a week following subculturing. The cell viability and confluence were checked regularly before reseeding. The drug was only given when the viability $>90 \%$ and the confluence $>50 \%$. Otherwise, the cell line was grown in drug-free media until the cells returned to confluence and the viability has recovered. Sensitivity to cisplatin was measured every 8 weeks using MTT-colorimetric test.

Healthy cells, which had been sub-cultured at least once and subsequently reached 70$80 \%$ confluency, were used for the MTT assay. Briefly, $100 \mu \mathrm{l}$ cell suspensions (105 cells $/ \mathrm{ml}$ ) were plated in 96-wells microplate. Cisplatin with raising concentration was added into the corresponding well; the control wells were left untreated. After 5 days incubation in $37{ }^{\circ} \mathrm{C}$ with $5 \% \mathrm{CO}, 30 \mu \mathrm{l}$ of MTT solution $(5 \mathrm{mg} / \mathrm{mL}$ ) was added into each well and the incubation was continued for a further 4 hours. Subsequently, $100 \mu \mathrm{L}$ DSO was added into each well to dissolve the formazan crystal. The optical density (OD) was measured using Multiskan FC $®$ (test wavelength of $590 \mathrm{~nm}$ and reference wavelength at $690 \mathrm{~nm}$ ). Cell viability was calculated using the following equation:

Cell viability (\%) = OD0/ODn x 100\%

Where $\mathrm{OD}_{0}$ is the OD from the control wells and ODn is the OD of the treated cells. The IC50 values were calculated as the concentrations that show $50 \%$ inhibition of cell.

The cell slides were prepared from cell suspension $\left(10^{5}\right.$ cells $\left./ \mathrm{mL}\right)$ and stained with hematoxylin-eosin staining. The morphological changes were observed to evaluate the signs of apoptosis, such as plasma membrane blebbing, condensation of chromatins, nuclear condensation, and formation of apoptotic bodies. $^{7}$

The statistical analyses were performed using Graphpad prism software version 5.0 (GraphPad Software, San Diego California USA). Data was presented as mean \pm standard mean error (SEM). The comparison of means was performed by using Student's t-test and ANOVA with Tukey multiple comparison as a post hoc analysis. P-value smaller than 0.05 was considered statistically significant.

\section{Results}

In this study, the MCF-7 resistance to cisplatin was developed in-vitro by introducing an increasing dose of cisplation, using a low startup dose $(0.01 \mu \mathrm{M})$. Cisplatin is known as one of several platinum based anticancer drugs which are widely used in cancer therapy and have demonstrated a successful outcome in breast cancer therapy. ${ }^{4,5,7}$ In this study, the parental cell line MCF-7 exerted sensitivity to cisplatin, demonstrated by a low $\mathrm{IC}_{50}$ value $(2.72 \pm 0.51$ $\mu \mathrm{M})$ (Fig. 1). The sensitivity towards cisplatin and high successful rate of cisplatin therapy is attributed to multiple molecular mechanisms in combating cancer cells, which is mediated by its ability to interact with deoxyribonucleic acid (DNA) strands causing DNA adduct. ${ }^{6,11,12}$ The DNA damage is recognised by several proteins as a signal to activate further pathways, including p53, ATR, and MAPK. The crosstalk between these pathways ultimately leads to cell cycle arrest, apoptosis and cell death. ${ }^{8}$

Despite the effectiveness of cisplatin as chemotherapy agent, cancer cells might have a natural ability to turn this cytotoxic effect, which might be a result from continuous drug exposure. ${ }^{4,5,9}$ In the establishment of the drug 

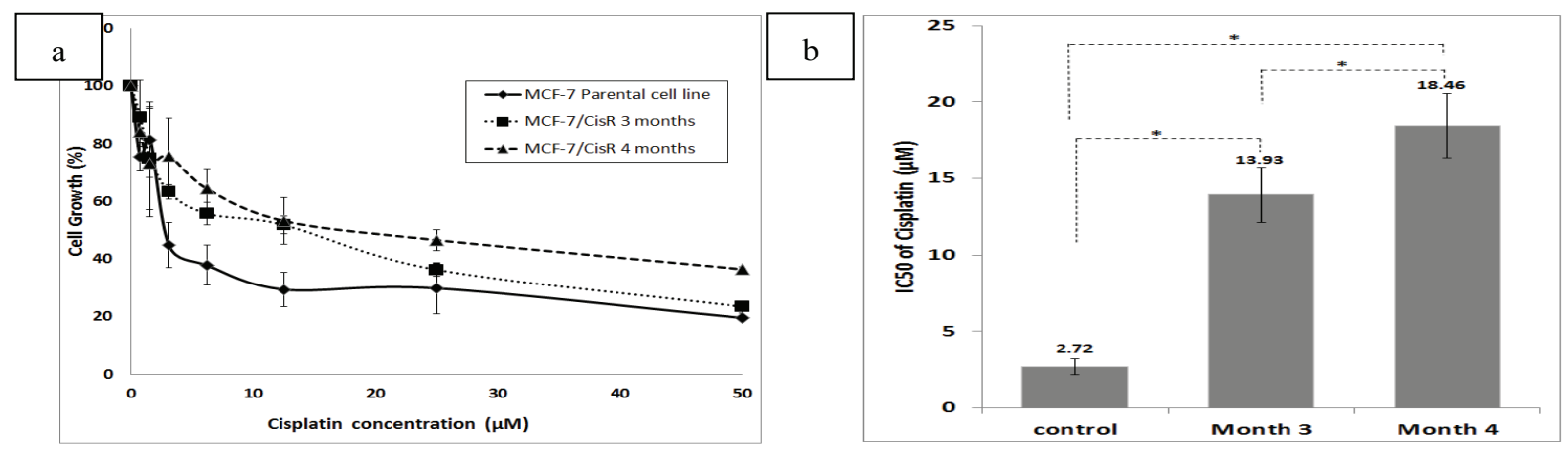

Fig. 1 Cell Growth Curve Showing the Percentage of MCF-7 Cell Growth againts a

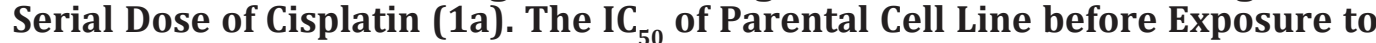
Cisplatin (Control), after 3 Months, and after 4 Months ((1b). For the Calculation of IC ${ }_{50}$ Value, the Largest Value in the Data Set Corresponded to $100 \%$ and the Smallest Value Corresponded to $0 \%$. Log-Transformed Drug Concentrations were Plotted against the Dose Response and the $\mathrm{IC}_{50}$ Values were Determined Using Non-Linear Regression Analysis ( $* \mathrm{P}<0.05$, ANOVA)

resistance on one particular cancer cell line, the mode of drug administration is a key factor that needs careful consideration. In a clinical setting, chemotherapy is given in cycles by intravenous administration on a weekly or monthly basis. ${ }^{10}$

The amount of chemotherapy agents given is based on individual needs and will be adjusted based on response to the chemotherapy; the dose is often expressed as milligrams per square meter unit which can be converted to micromolar $(\mu \mathrm{M})$ unit. ${ }^{11}$ In this study, the parental line was carefully observed during the cisplatin-resistance development process by conducting evaluation to the viable cells before increasing the cisplatin dose. This method was applied to mimic the clinical treatment, where the response to chemotherapy was reduced/ increased based on the patient response. The range of cell viability was maintained between $93-100 \%$, in order to ensure that the cells were growing in an optimum condition and able to escape the toxic effect of cisplatin.

After 3 months treatment, the MCF-7/CisR cell line has developed a significant resistance, demonstrated the $\mathrm{IC}_{50}$ value which was more than 5 times compared to parental cell $\mathrm{IC}_{50}$ value $(13.93 \pm 1.8 \mu \mathrm{M})$. When the treatment was continued, the $\mathrm{IC}_{50}$ value rose to $18.46 \pm 2.1$ $\mu \mathrm{M}$ (almost 7 times parental cell $\mathrm{IC}_{50}$ ). At this stage, the cisplatin-resistant phenotype was established in-vitro.
Although there is no clear-cut value for the resistance level of cisplatin therapy, previous clinical studies have suggested that at least twofold resistance can be considered as resistance in chemotherapy. ${ }^{4}$ This present study has successfully developed acquired resistance on the MCF-7 cell line, noted as cisplatin resistant subline MCF-7/CisR. Drug resistance is the condition where cisplatin was initially efficient to kill the cancer cells, but became ineffective over time following continuous drug exposure. ${ }^{5}$

The resistance mechanism is believed as a net effect from intracellular changes that inhibit cisplatin interaction with DNA, and/ or alter the transduction of DNA damage signal to activate the apoptotic pathways. ${ }^{9,12}$ The former mechanism arises form direct correlation between the level of DNA adduct with the cytotoxicity and, therefore, reducing the extent of DNA damage increases cisplatin resistance. ${ }^{4,9}$ Other intracellular mechanisms which have a correlation to acquired resistance to cisplatin include a reduction of intracellular cisplatin accumulation, increase inactivation of cisplatin (detoxification) via gluthationmediating intoxication pathway, and improved repair of and/or tolerance to nuclear lesions/ DNA damage. ${ }^{4}$ All these mechanisms work together simultaneously to develop and maintain cancer cell resistance to cytotoxic agents. ${ }^{4,12,14,17}$ 

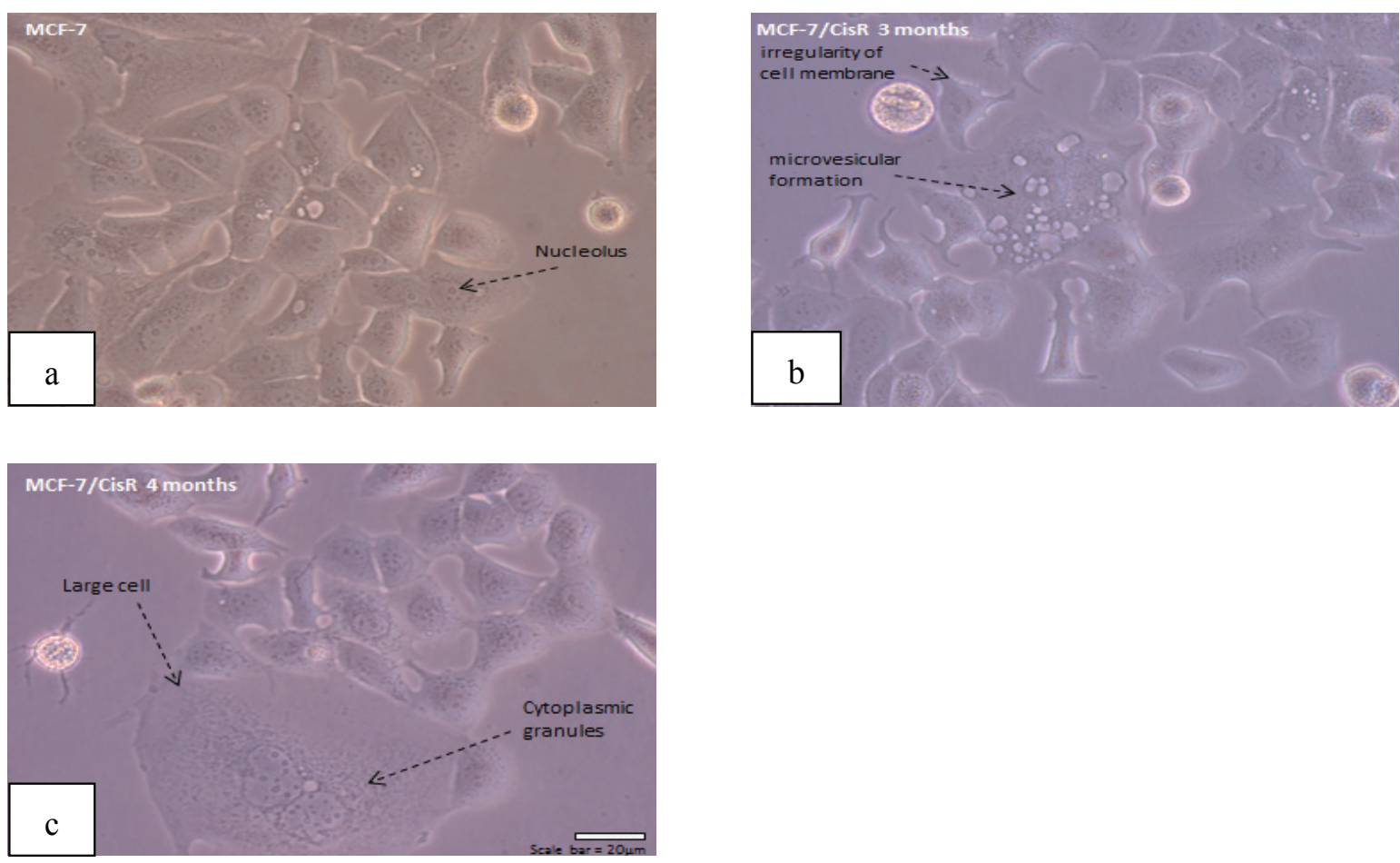

Fig. 2 Microscopic Observation of MCF-7 Parental Cells, MCF-7/CisR after 3 Months and 4 Months of Cisplatin Exposure (at Magnification 400x). MCF-7 Parental Cell Line Shows a Regularity in Shape and Size, with 2-5 Nucleoli (2a). After 3 Months Treatment with Cisplatin, Cell Edge Became Irregular, Accompanied by Irregularity of the Plasma Membrane and Microvesicular Formation. After the MCF-7/CisR was Established on the 4th Month of Cisplatin Treatment, Large Cells were Seen, with the Increase of Cytoplasmic Granules (2c)

\section{Discussion}

The change of the proliferation potential on MCF-7 resistant subline MCF-7/CisR is an ultimate effect of molecular mechanisms by which the cancer cells obtain an ability to avoid the cytotoxic effect of anti-cancer drugs. Accumulating evidence suggests that breast cancer cell resistance is strongly related to several theories, namely the existence of cancer stem cells (CSCs) which is marked by the increased of CSCs markers including CD44+/CD24-, high level of phosphorylated Stat-3 and its downstream effector proteins, and the occurance of breast cancer resistance protein (BCRP). ${ }^{13}$ More importantly, all these molecular and phenotypic modifications on one cell are often accompanied by changes in morphological features. ${ }^{11}$ This current study observed importantmorphological parameters of MCF-7 cells following continuous treatment with cisplatin; including cell shape, cell size and cell appearance.

In a normal environment, regulation of cell size is differentiated into two distinctprocesses, namely cell growth and cell proliferation. Both processes can be independent of each other as one cell can grow without cell proliferation, and vice versa. ${ }^{14}$ Several growth pathways are responsible for controlling cell proliferation and cell growth, for example, the PI3K/ AKT pathway, which is noted as the major determinant and major regulator of cell size. ${ }^{14-}$ ${ }^{16}$ Cancer cells are often developed through the loss of control to maintain normal cell growth and proliferation rate, as the result from the alteration of these growth pathways. As the hallmark of cancer cells is able to escape 
programmed cell death and possess limitless proliferation potential, it is not surprisingly, the cells are able to maintain their intrinsic signal to survive any unfavourable external stimulus given. In their effort to escape the deadly effect of chemotherapy drugs, cancer cells must be able to modify their intrinsic developmental program, in a way that makes the extracellular signals are less harmless to the cell growth and proliferation.

The result showed that, according to the relative size of the cells, parental cells MCF-7 have a relatively similar size (20-25 microns), whereas resistant cell MCF-7/CisR population comprised a wider range of cell size (20 microns up to more than 50 microns in diameter). MCF-7/CisR cells have a larger nucleus, although the nucleolus number is similar to the parental cell line (2-5 nucleoli). The variation of the cell size is determined by the intrinsic developmental program and by extracellular signals, where the combination of these factors will integrate to control cell number and cell proliferation. ${ }^{14}$ Irregularity of the cell shape and nuclear enlargement showed that cells within the population of MCF-7/ CisR were in various stages of the cell cycle. During the cell cycle, there are two important checkpoints, G1 and G2, which simultaneously search for the occurrence of DNA damage and determine whether the cell can proceed to the next cell cycle stage or not. ${ }^{15}$ When DNA damage is detected, the cells are arrested for DNA repair or, when the damage is irreversible, checkpoint effector mechanisms will activate the so-called self-destruction mechanism or apoptosis. ${ }^{15,16}$ Cancer cells possess an ability to avoid this checkpoint mechanism and to escapes apoptosis. ${ }^{17}$ Cisplatin therapy is aiming at the re-activation of the apoptosis cascade by inducing DNA damage, due to its ability to crosslink with the purine bases of the DNA. ${ }^{6,7}$

Cisplatin binding to cancer cell's DNA causes DNA adduct, which is recognised by DNA damage recognition proteins, for example p53 protein and its affector proteins. ${ }^{5,6}$ In the case of cisplatin resistance, some cells might survive the cytotoxic effect of cisplatin, causing the resistant cells to grow and to proliferate at a different state within the cell cycle and, accordingly, demonstrate a wide range of cell sizes at one point of time, as shown in our result. In agreement with the results in this study, some reports have suggested that cisplatin resistance on MCF-7 involves antiapoptotic mechanisms, demonstrated by a decrease of DNA-damage effector proteins, which is strongly related to the ability of the resistant cells to tolerate and repair the DNA damage due to cisplatin activity.5,6,18

In this present study, we demonstrated that in comparison to its parental cell line MCF-7, MCF-7/CisR demonstrated several observable morphological changes. Parental cell line MCF-7 exhibited a regular spindle shape with a smooth cell membrane border, while the resistant cells demonstrated an irregular and spherical shape where some parts or the cell border are unclear (Fig. 2). The result is in line with other research in cancer chemoresistance. One study on colon cancer (using HCT 116 cell lines) conducted by Pasqualato et $a l^{20}$ demonstrated a significant change in cell membrane following continuous treatment with anticancer drugs, accompanied by the alteration of other biological parameters. The studies also argued that the changes of cell morphology would be an indicator of chemoresistance, thereby marking the level of malignancy. ${ }^{19,20}$ In line with previous reports, the result in this study has demonstrated an insight on the relationship between phenotype and morphological changes in MCF-7 cisplatin resistance.

The result in this study showed that the number or microvesicular and cytoplasmic granules increased significantly in MCF-7 resistant cells (Fig. 2). The increase of the microorganelles might be related to the molecular transport from intracellular to extracellular compartment. The microvesicular formation indicates cell activity to increase drug efflux from the cytoplasm, to reduce net concentration of cisplatin in the cells.

There is a considerable number or evidence indicating a significant contribution of the reduction of intracellular drug accumulation in the cisplatin resistance mechanism. ${ }^{7,9,12}$ Although many questions still remain, which stem from the multifactorial nature of drug uptake and drug efflux processes. For example, in the case of MCF-7 resistance to cisplatin, a study reported that exporter proteins, for example P-glycopotein, are less likely to be involved in the cisplatin chemoresistance. ${ }^{5}$ Furthermore, a particular number of evidence has suggested that there is an extensive change of the ultrastructural organization in resistant cell lines, including extensive golgi apparatus with many multivesicular bodies, the increase on the number of microtubules and microfilaments, and a significant rise of the mitochondria number. ${ }^{5,6}$

A previous study revealed that the resistance to cisplatin is directly related to the 
hyperactivity of mitochondria in performing oxidative phosphorylation, which can increase the intracellular metabolism rate. ${ }^{19}$ Moreover, growing evidence has shown that significant morphological changes is also linked to cell energy states. ${ }^{19}$ With an excessive energy production, the resistant cell lines are able to proceed with DNA repair and expelled cisplatin from the intracellular environment. Therefore, this present study might explain that the mechanism of cisplatin efflux is more likely controlled by cellular metabolic activity, rather than drug transporter activity, which ultimately decreases cisplatin accumulation within the cells. Despite the limitation of our study, which restrains the detailed observation of cell morphology, our result has provided a vibrant supporting evidence for further confirmation of microorganelles changes as a results from molecular mechanism of cisplatin resistance in MCF-7 cell line.

In conclusion, different therapies have been established for combating breast cancer. However, drug resistance is still counted as a major drawback in cancer treatment, particularly in cancer chemotherapy.

Cisplatin, although known as one of effective chemotherapy agents for breast cancer, frequently confronts the problem of resistance. This study demonstrated that morphological changes occur in MCF-7 cisplatin resistant cells, including the enlargement of cell size, the increase of nucleous and cytoplasm ratio, the increase of the number of microvesicular and cytoplasmic granules. The result supported the previous studies which have indicated that the cisplatin-resistance mechanism is related to the ability of the cells to decrease intracellular cisplatin concentration and to repair the effects of DNA damage. Nevertheless, there are major gaps that need to be filled to fully understand the clear links between molecular mechanisms and morphological changes of MCF-7 resistant phenotype.

\section{References}

1. Gonzalez-Angulo AM, Morales-Vasquez F, Hortobagyi GN. Overview of resistance to systemic therapy in patients with breast cancer. Adv Exp Med Biol. 2007;608(1):1-22.

2. Sun $H$, Jia J, Wang $X$, Ma B, Di L, Song G, et al. CD44+/CD24- breast cancer cells isolated from MCF-7 cultures exhibit enhanced angiogenic properties. Clin Translational Oncol. 2013;15(1):46-54.

3. WHO. Breast cancer: prevention and control. [cited 2011 Nov 1]. Available from: http://www.who.int/cancer/detection/ breastcancer/en/index1.html.

4. Florea AM, Büsselberg D. Breast cancer and possible mechanisms of therapy resistance. J Local Global Health Sci. 2013;2(2):2.

5. Lukyanova NY, Rusetskya NV, Tregubova NA, Chekhun VF. Molecular profile and cell cycle in MCF-7 cells resistant to cisplatin and doxorubicin. Exp Oncol. 2009;31(2):87-91.

6. Meijera C, van Luyn MJA, Nienhuis EF, Blom N, Mulder NH, de Vries EGE, et al. Ultrastructural morphology and localisation of cisplatininduced platinum-DNA adducts in a cisplatinsensitive and -resistant human small cell lung cancer cell line using electron microscopy. Biochem Pharmacol. 2001;61(5):573-8.

7. Dasari S, Tchounwou PB. Cisplatin in cancer

therapy: molecular mechanisms of action. Eur J Pharmacol. 2014;5(1):364-78.

8. Cepeda V, Fuertes MA, Castilla J, Alonso C, Quevedo C, Pérez JM. Biochemical mechanisms of cisplatin cytotoxicity. Anti-Cancer Agents Medicinal Chem. 2007;7(1):3-18.

9. Siddik ZH. Cisplatin: mode of cytotoxic action and molecular basis of resistance. Oncogene. 2003;22(47):7265-79.

10. Byrski T, Huzarski T, Dent R, Marczyk E, Jasiowka M, Gronwald J, et al. Pathologic complete response to neoadjuvant cisplatin in BRCA1-positive breast cancer patients. Breast cancer research and treatment.2014;147(2): 401-5.

11. McDermott M, Eustace AJ, Busschots S, Breen L, Crown J, Clynes M, et al. In vitro development of chemotherapy and targeted therapy drugresistant cancer cell lines: a practical guide with case studies. Frontier Oncol. 2015;4(40):3248.

12. Rebucci M, Michiels C. Molecular aspects of cancer cell resistance to chemotherapy. Biochem Pharmacol. 2013;85(9):1219-26.

13. Mao Q, Unadkat JD, Role of the (BCRP/ ABCG2) in drug transport--an update. AAPS J. 2015;17(1):65-82.

14. Lloyd AC. The regulation of cell size. Cell. 
2013;154(6):1194-205.

15. Dickson MA, Schwartz GK. Development of cell cycle inhibitors for cancer therapy. Curr Oncol. 2009;16(2):36-43.

16. Bucher N, Britten C. G2 checkpoint abrogation and checkpoint kinase-1 targeting in the treatment of cancer. $\mathrm{Br} \mathrm{J}$ Cancer. 2008;98(3):523-8.

17. Lazebnik Y. What are the hallmarks of cancer? Nat Rev Cancer. 2010;10(4):232-33.

18. LaPensee EW, Ben-Jonathan N. Novel roles of prolactin and estrogens in breast cancer: resistance to chemotherapy. Endocrine-related Cancer. 2010;17(2): R91-107.

19. D’Anselmi F, Valerio M, Cucina A, Galli L, Proietti S, Dinicola S, et al. Metabolism and cell shape in cancer: a fractal analysis. Int J Biochem Cell Biol. 2011;43(7):1052-8.

20. Pasqualato $A$, Palombo $A$, Cucina $A$, Mariggiò MA, Galli L, Passaro D, et al. Quantitative shape analysis of chemoresistant colon cancer cells: correlation between morphotype and phenotype. Exp Cell Res. 2012;318(7):835-46. 